\title{
Association between cancer stem cell-like properties and epithelial-to-mesenchymal transition in primary and secondary cancer cells
}

\author{
WONBONG LIM ${ }^{1 *}$, HYE-EUN KIM ${ }^{2 *}$, YOUNG KIM ${ }^{2}$, RISU NA ${ }^{2}$, XIAOJIE LI $^{3}$, \\ SANGMI JEON ${ }^{2}$, HONGRAN $\mathrm{CHOI}^{2}$ and OKJOON KIM ${ }^{2}$
}

\author{
${ }^{1}$ Department of Premedical Science, College of Medicine, Chosun University, Dong-Gu; ${ }^{2}$ Department of Oral Pathology, \\ Dental Science Research Institute and Medical Research Center for Biomineralization Disorders, \\ School of Dentistry, Chonnam National University, Bug-Gu, Gwangju, Republic of Korea; \\ ${ }^{3}$ Stomatology College of Dalian Medical University, Dalian, Liaoning 116044, P.R. China
}

Received April 15,2016; Accepted June 6, 2016

DOI: 10.3892/ijo.2016.3582

\begin{abstract}
One of the theories on cancer stem cells (CSCs) states that these cells initiate most tumors and give rise to more-or-less differentiated tumor cells. Genetic signatures of CSCs are thought to predict tumor recurrence and metastases, thus, supporting the notion that CSCs may be metastatic precursors and induce epithelial-to-mesenchymal transition (EMT). In this study, we tried to examine the association between CSCs and EMT (using specific markers) in the mucoepidermoid carcinoma cell line YD15 and its derivative cell line YD15M (lymph node metastasis). Relative protein expression levels were analyzed by western blotting, flow cytometry, and immunofluorescence assays. In addition, cell cycle assay and aldehyde dehydrogenase (ALDH) activity assay were carried out. Under growth conditions, YD15M cells formed irregular spherical colonies consistent with a stem cell phenotype. YD15M cells demonstrated the low expression of E-cadherin and $\beta$-catenin but high expression of vimentin than that in YD15 cells. In the metastatic cells (YD15M), the coexpression of vimentin and CD133 was detected. Weak proliferation based on cell cycle analysis and decreased PCNA expression was also observed. In addition, expression levels of ALDHA1, OCT4, and NANOG (CSC-like properties) were significantly increased in YD15M cells. Taken together, these findings should help to elucidate the interplay between EMT
\end{abstract}

Correspondence to: Professor Okjoon Kim, Department of Oral Pathology, Dental Science Research Institute and Medical Research Center for Biomineralization Disorders, School of Dentistry, Chonnam National University, Bug-Gu, Gwangju 500-757, Republic of Korea E-mail: js3894@chonnam.ac.kr

*Contributed equally

Key words: CD133, cancer stem cell, carcinogenesis, epithelial-tomesenchymal transition and CSC-like properties during metastasis and may provide useful information for the development of a novel classification system and therapeutic strategies against head and neck cancer.

\section{Introduction}

Oral cancer is the most prevalent type of cancer in the oral cavity and is associated with high mortality and poor prognosis $(1,2)$. Despite surgical treatment, chemotherapy, or radiotherapy, the 5-year survival rate has remained $<50 \%$ in the past decades (3). Recurrence and distant metastases play a crucial role in the morbidity and mortality associated with oral cancer, and their mechanisms remain unclear (4). During progression to metastases, tumor cells losing their adhesive ability become detached from the primary tissue, invade the basement membrane, circulate via blood vessels, and finally colonize a new location in the host. A growing body of evidence suggests that the epithelial-to-mesenchymal transition (EMT), which occurs normally during embryonic development, tissue remodeling, and wound healing, is a critical early event in metastasis (4-6). EMT is the cellular and molecular process by which cell-to-cell interactions and apicobasal polarity disappear and a mesenchymal phenotype develops; these events are required for cell motility and invasion of the basement membrane during metastasis $(7,8)$. Furthermore, researchers have shown that EMT is associated with the dedifferentiation program that leads to malignant carcinoma, because EMT is responsible for the ability of invasive cancer cells to migrate and reach distant tissues (9).

Several research groups have studied cancer stem cells (CSCs) during metastasis and found that these cells are associated with tumor recurrence after treatment and with treatment resistance $(10,11)$. CSCs, as defined by the American Association for Cancer Research, are a small minority of cells with the capability of self-renewal and differentiation into the heterogeneous lineages that proliferate extensively and constitute the tumor mass $(12,13)$. This minority of cancer cells is believed to be responsible for formation of the bundle of a 
tumor that consists of cells at varying stages of differentiation (14). In this respect, the hierarchy of a tumor is similar to the tissue from which it originates, and CSCs are considered neoplastic counterparts of normal stem cells. Thus, CSCs are expected to have a stem cell-like phenotype (generally referred to as stemness). Furthermore, CSCs have been found in several types of solid tumors, such as ovarian (15), brain $(16,17)$, breast $(18)$, prostate (19), and pancreatic cancers (11) as well as melanoma (20) and head and neck cancer (21-23).

A recent breakthrough in EMT research showed that EMT can impart stem cell-like properties of CSCs to epithelial cells. The acquisition of stem cell-like properties by cancer cells is a crucial step for cancer progression and treatment resistance (23). Some mechanisms underlying the EMT-induced stem cell-like properties of cancer cells have been uncovered. Epithelial cells undergoing EMT are characterized by the downregulation of epithelial makers (such as cytokeratin; CK), a loss of cell polarity, and intercellular adhesion molecules (for instance E-cadherin and occludin), which is accompanied by the upregulation of mesenchymal markers (vimentin, $\mathrm{N}$-cadherin, and fibronectin), and acquisition of fibroblast-like morphological features with cytoskeleton reorganization. The loss of E-cadherin and acquisition of $\mathrm{N}$-cadherin are known as cadherin switching, a major hallmark of EMT (24). The role of adhesion molecules in EMT-induced stemness in CSCs remains unclear.

Because CD133 (PROM1) is expressed by hematopoietic progenitors, it aroused great interest regarding its potential as a cell surface marker of CSCs (14). The CD133 protein is a pentaspan cell surface receptor; neither its ligand nor its secondary messengers are known. According to one study, CD133 is expressed in combination with the $\mathrm{CD} 44^{+} \alpha 2 \beta 1^{\text {high }}$ phenotype in $\sim 0.1 \%$ of cells in a large number of samples of prostate tumors, irrespective of their grade or metastatic state (25). These cells are capable of self-renewal, proliferation, and multilineage differentiation in vitro, with recapitulation of the original tumor phenotype, consistent with CSC properties $(19,26)$. Olempska et al showed that ABCG2 and CD133 are strongly coexpressed in two out of five human pancreatic adenocarcinoma cell lines; thus, CD133 may also be a putative CSC marker in the pancreas (27). CSCs are significantly enriched in $\mathrm{CD}_{133^{+}}$subpopulations of human colon cancer cells and hepatocellular carcinomas, according to the ability of these $\mathrm{CD}_{133^{+}}$cells to self-renew, differentiate, and to form colonies and proliferate in vitro and judging by their ability to modify the original tumor phenotype when tumor-derived $\mathrm{CD}_{133^{+}}$cells are transplanted subcutaneously into immunodeficient mice (28). Growing evidence suggests that EMT bestows carcinoma cells at the tumor front with CSC-like properties and plays an important role in formation of CSCs. Nevertheless, no one has studied the possible association of EMT specification with CSC-like properties associated with CD133 expression in primary cancer cells and metastatic cells.

On the basis of the above studies, here, we demonstrate in oral-cancer cells the molecular mechanisms that are involved in the expression of CD133 by the cancer cells: the mechanisms that induce EMT and lead to acquisition of CSC-like properties by primary tumor cells and metastatic cells of head and neck cancer. These results may point to new avenues of research with clinical implications.

\section{Materials and methods}

Cell culture. Experiments were performed on two cell lines: YD15 and YD15M. YD15 cells were derived from mucoepidermoid carcinoma of the tongue and YD15M cells from a lymph node metastasis from YD15. Both cell lines were purchased from the Korean Cell Line Bank (KCLB, Seoul, Korea; cat. nos. 60504 and 60505, respectively) and maintained in the RPMI-1640 medium (Gibco, Daejeon, Korea) supplemented with $10 \%$ heat-inactivated fetal bovine serum (Biomeda Co., CA, USA) and 10\% antibiotic-antimycotic solution (Welgene, Daegu, Korea) at $37^{\circ} \mathrm{C}$ in a humidified atmosphere containing $5 \%$ of $\mathrm{CO}_{2}$. The medium was replaced with a fresh one, and the adherent cells were allowed to reach $\sim 70 \%$ confluence. Then, the cells were detached by means of a trypsin-ethylenediamine tetraacetic acid solution (trypsinEDTA: Gibco-BRL) and plated again (subcultured) in 6-well plates for each experiment.

Western blotting. When the cells reached $\sim 70 \%$ confluence, the medium was removed, and the cells were washed twice with phosphate-buffered saline (PBS, $\mathrm{pH} 7.4)$. Then, the cell lysate was prepared in $200 \mathrm{ml}$ of cold lysis buffer $(1 \% \mathrm{NP}-40,50 \mathrm{mM}$ Tris- $\mathrm{HCl} \mathrm{pH} 7.5,150 \mathrm{mM} \mathrm{NaCl}, 0.02 \%$ sodium azide, $150 \mathrm{mg} /$ $\mathrm{ml}$ phenylmethylsulfonyl fluoride (PMSF), $2 \mathrm{mg} / \mathrm{ml}$ aprotinin, $20 \mathrm{mg} / \mathrm{ml}$ leupeptin, and $1 \mathrm{mg} / \mathrm{ml}$ pepstatin A). Approximately $30 \mathrm{mg}$ of protein of the cell lysate was separated by sodium dodecyl sulfate polyacrylamide gel electrophoresis (in a $10 \%$ gel) and transferred to a polyvinylidene difluoride membrane (Amersham, Piscataway, NJ, USA). Each membrane was blocked with a blocking solution containing 5\% skim milk in Tris-buffered saline with Tween-20 (TBST; 2.42 g/l Tris-HCl $\mathrm{pH} 7.6,8 \mathrm{~g} / 1 \mathrm{NaCl}, 0.1 \%$ Tween-20) for $0.5 \mathrm{~h}$ and rinsed briefly in TBST. The membrane was incubated overnight at $4^{\circ} \mathrm{C}$ with the appropriate primary antibody: an anti-E-cadherin antibody (dilution 1:1,000, Santa Cruz Biotechnology, Santa Cruz, CA, USA), anti- $\beta$-catenin (1:1,000, Santa Cruz Biotechnology), anti-vimentin (1:1,000, Santa Cruz Biotechnology), anti-CD133 (1:500, Mybiosource, San Diego, CA, USA), anti-PCNA (1:1,000, Cell Signaling Technology, Beverley, MA, USA), antipanCK (1:1000, Cell Signaling Technology), anti-ALDHA1 (1:1,000, Cell Signaling Technology), anti-OCT-4 (1:1,000, Cell Signaling Technology), or an anti-NANOG antibody (1:1,000, Cell Signaling Technology). A mouse monoclonal anti- $\beta$-actin antibody (1:2,500, Santa Cruz Biotechnology) was used as the control. Finally, the membrane was washed in TBST and the immunoreactivity of the proteins was analyzed with an enhanced chemiluminescence detection kit (Santa Cruz Biotechnology). The intensity of bands was quantified in densitometric software ImageJ (http://rsb.info.nih.gov/ij).

Double immunofluorescence staining and confocal microscopy. The immunofluorescence assay was performed as described previously (29). Cells were seeded onto glass Lab-Tek II Chamber Slides (Thermo Fisher Scientific Inc., Loughborough, UK) and incubated for 1 day. The growth medium was then removed, and cell monolayers were washed three times with a $1 \%$ PBS solution and fixed with $3.5 \%$ paraformaldehyde for $10 \mathrm{~min}$ at room temperature. The cells were washed three times with PBS and permeabilized by Triton 
$\mathrm{X}-100(0.2 \%, 10 \mathrm{~min}$ at room temperature). Non-specific binding sites were blocked by incubation with PBS containing $1 \% \mathrm{BSA}$, for $30 \mathrm{~min}$ at room temperature. The cells were washed three times with PBS before incubation with the rabbit anti-vimentin antibody (1:200) followed by a fluorescein isothiocyanate (FITC)-conjugated goat anti-rabbit IgG antibody $(1: 1,000)$ and a mouse anti- $\beta$-catenin antibody (1:200; or a mouse anti-CD133 antibody, 1:200), with subsequent incubation with a Texas Red (TR)-conjugated goat anti-mouse IgG antibody $(1: 1,000)$. In some cases, the samples were incubated with the rabbit anti-PCNA antibody (1:200) followed by the FITC-conjugated goat anti-rabbit IgG antibody $(1: 1,000)$ and mouse anti-CD133 antibody (1:200) with subsequent incubation with the TR-conjugated goat anti-mouse IgG antibody $(1: 1,000)$. The cells were counterstained with 4',6-diamidino-2-phenylindole (DAPI) for visualization of nuclear morphology. The stained cells were analyzed under a confocal microscope (Carl Zeiss, Oberkochen, Germany), at excitation wavelength $490 \mathrm{~nm}$ and emission wavelength $540 \mathrm{~nm}$ for FITC, excitation $540 \mathrm{~nm}$ and emission $650 \mathrm{~nm}$ for TR, and excitation $360 \mathrm{~nm}$ and emission $450 \mathrm{~nm}$ for DAPI.

Flow cytometric assessment of protein expression. Suspensions of YD15 and YD15M cells that were detached with trypsin-EDTA were washed with PBS and fixed with $3.5 \%$ paraformaldehyde for $10 \mathrm{~min}$ at room temperature. The cells were washed three times with PBS and permeabilized by Triton X-100 (0.2\%, $10 \mathrm{~min}$ at room temperature). Non-specific binding sites were blocked by treatment with PBS containing 1\% BSA, at room temperature for $30 \mathrm{~min}$. The cells were washed three times with PBS before incubation with the rabbit anti-vimentin antibody $(1: 1,000)$ followed by the FITC-conjugated goat anti-rabbit IgG antibody $(1: 1,000)$ and mouse anti-CD133 antibody $(1: 1,000)$ with subsequent incubation with the TR-conjugated goat anti-mouse IgG antibody $(1: 1,000)$. In some cases, the samples were incubated with the rabbit anti-PCNA antibody $(1: 1,000)$ followed by the FITC-conjugated goat anti-rabbit IgG antibody $(1: 1,000)$ and mouse anti-CD133 antibody $(1: 1,000)$ with subsequent incubation with the TR-conjugated goat anti-mouse IgG antibody $(1: 1,000)$. After the incubation, the cells with the bound antibodies were washed twice with washing buffer and analyzed by flow cytometry (Beckman Coulter, CA, USA). Approximately 10,000 counts were accumulated for each sample. Anti-IgG (isotype) antibodies were used to assess the non-specific binding (background) in this assay.

Magnetic cell separation. Magnetic cell separation (MACS) of vimentin ${ }^{+}$cells was performed with EasySep ${ }^{\mathrm{TM}}$ magnet according to the manufacturer's instructions (Stem Cell Technologies, Durham, NC, USA). Briefly, YD15 and YD15M cells were plated in 6-well plates. After they reached $70 \%$ confluence, the cells were detached and washed twice in cold PBS. Dissociated cells were stained with the rabbit antivimentin antibody $(1: 1,000)$ followed by the FITC-conjugated goat anti-rabbit IgG antibody $(1: 1,000)$ and were incubated for $30 \mathrm{~min}$ at $4^{\circ} \mathrm{C}$. After incubation with immunomagnetic microbeads for $30 \mathrm{~min}$ at $4^{\circ} \mathrm{C}$, the cells were washed in PBS containing $0.5 \% \mathrm{BSA}$ and $2 \mathrm{mM}$ EDTA, filtered through a 40-mm cell strainer. Then the cells were passed through the magnetic cell separation device (Auto-Macs; Miltenyi Biotec) for selection of vimentin positive cells.

Flow cytometric analysis of cell cycle with propidium iodide (PI) staining. Cells were trypsinized to obtain a single-cell suspension, harvested by centrifugation, and washed with PBS. After fixation in ice-cold $70 \%$ ethanol at $4^{\circ} \mathrm{C}$ overnight, the cells were collected and washed twice with PBS. The cells were then stained with PI $(3 \mu \mathrm{g} / \mathrm{ml})$ for $15 \mathrm{~min}$. After that, DNA from $10^{4}$ of G0/S phase cells was quantified on the flow cytometer (Beckman Coulter).

Flow cytometric assessment of ALDH activity. This assay of tumor-derived cells was conducted using the ALDEFLUOR kit (StemCell Technologies). In particular, the cells were suspended in ALDEFLUOR assay buffer containing ALDH1 substrate (BODIPY-aminoacetaldehyde, $1 \mathrm{M}$ per $10^{6}$ cells) and incubated for $30 \mathrm{~min}$ at $37^{\circ} \mathrm{C}$. As a negative control, some batches of the cells were incubated with a specific ALDH1 inhibitor, diethylaminobenzaldehyde (DEAB; $50 \mathrm{mM}$ ). After that, the cells were washed twice with washing buffer and analyzed by flow cytometry (Beckman Coulter). Approximately 10,000 counts were accumulated for each sample.

Statistical analysis. The data were expressed as mean \pm standard deviation. All experiments were repeated three times, and all calculations were performed in Microsoft Excel. For significance testing, we used Student's t-test (at $\mathrm{P}<0.05$ ).

\section{Results}

The ability of primary cancer cells and metastatic cells to undergo EMT. The metastatic cell line, YD15M, which was derived from mucoepidermoid carcinoma, showed morphological features of cells undergoing EMT (Fig. 1A). The observed morphological changes in YD15M cells include switching from a cuboid epithelial shape to a fibroblastic elongated appearance, with colonies of irregular spherical shape. The protein expression analysis of YD15M cells revealed a loss of the epithelial markers E-cadherin and $\beta$-catenin; this was not the case in YD15 cells (Fig. 1B). Simultaneously, an increased level of the mesenchymal marker vimentin was observed in YD15M cells. To verify these findings, we used laser scanning confocal fluorescence microscopy to analyze the expression of these epithelial and mesenchymal markers (Fig. 1C). As shown in the figure, YD15M cells showed decreased protein expression of $\beta$-catenin (red) and increased expression of vimentin (green). Moreover, a small population of cells with nuclear localization of $\beta$-catenin was observed among YD15M cells, but not among YD15 cells, indicating that the metastatic YD15M cells were derived from YD15 cells in association with EMT.

Relation of CD133 with vimentin expression on primary cancer cells and metastatic cells. The protein expression levels of vimentin and CD133 were significantly higher in YD15M cells than in YD15 cells according to immunofluorescence assays (Fig. 2A). The percentage of cells expressing CSC-specific protein CD133 was greater among YD15M cells than among 


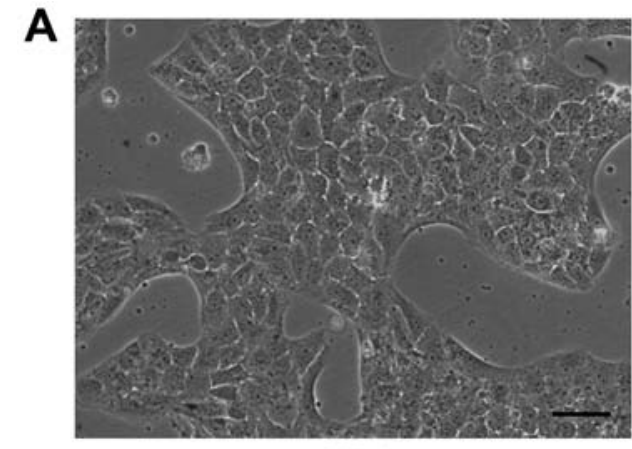

YD15

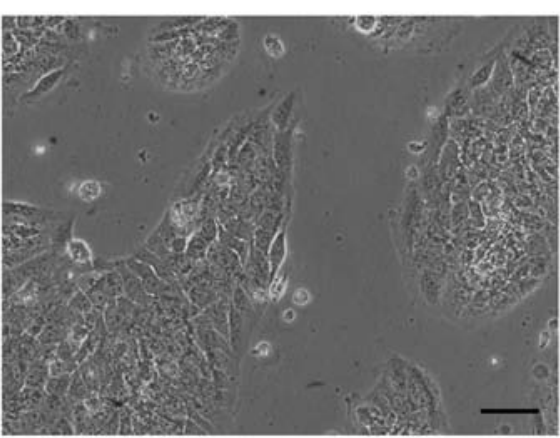

YD15M

\section{B}
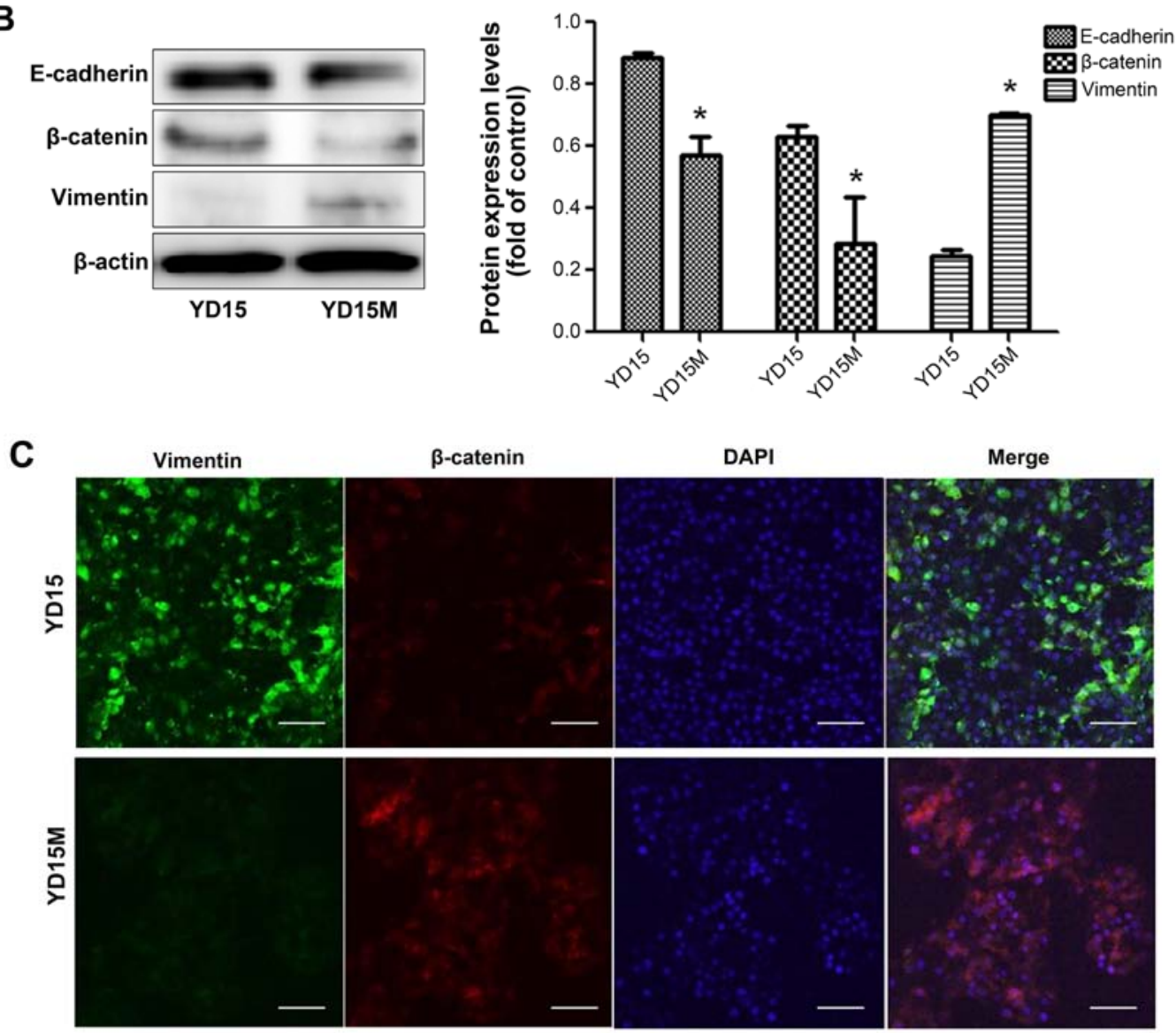

Figure 1. The ability of the primary cancer cell line YD15 and metastatic YD15M cells to undergo epithelial-to-mesenchymal transition (EMT) including the morphological changes. (A) The cell line YD15 possesses an epithelium-like phenotype, whereas YD15M cells form elongated colonies of irregular spherical shape. Images were taken under an inverted microscope (Olympus). The scale bars are $100 \mu \mathrm{m}$. (B) Expression of EMT-related proteins was characterized by western blot analysis. $\beta$-actin served as a loading control. The results were similar in 3 separate experiments and are presented as mean \pm SD (densitometric analysis, as shown in the graphs). ${ }^{*} \mathrm{P}<0.05$, significant differences. (C) Double immunofluorescence staining of vimentin (green) and $\beta$-catenin (red). The nuclei in both image sets were stained with 4',6-diamidino-2-phenylindole (DAPI; blue); x100 magnification. The scale bars are $100 \mu \mathrm{m}$.

YD15 cells. The CD133 protein was observed mainly in the cytoplasm and plasma membrane of the cells. To evaluate the correlation of vimentin and CD133 expression in cells of the primary and secondary tumors, we conducted dual-color flow cytometric analysis. As evident in Fig. 2B, the subpopulation of double-stained vimentin ${ }^{+} \mathrm{CD} 133^{+}$cells (gated in sector B2 in the graphs) constituted $1.8 \%$ of YD15 cells. In contrast, the percentage of cells in sector B2 among YD15M cells was much greater: $16.9 \%$; this result indicated that the metastasis associated with the double-stained $\mathrm{CD} 133^{+}$vimentin ${ }^{+}$cells showing features of EMT. To test whether the metastatic cells gained CSC-like properties, CD133 expression was assessed in vimentin ${ }^{+}$cells during MACS (Fig. 2C). As expected, the percentage of vimentin ${ }^{+} \mathrm{CD} 133^{+}$cells (gated in sector B2) was significantly greater $(20.0 \%)$ among YD15M cells than among YD15 cells $(5.7 \%)$. This finding means that the population of vimentin ${ }^{+}$cells acquired some properties of CSCs during metastasis.

The relation of proliferative characteristics with CSC marker expression on primary cancer cells and metastatic cells. We then tested whether the metastatic cells have the proliferative 
A
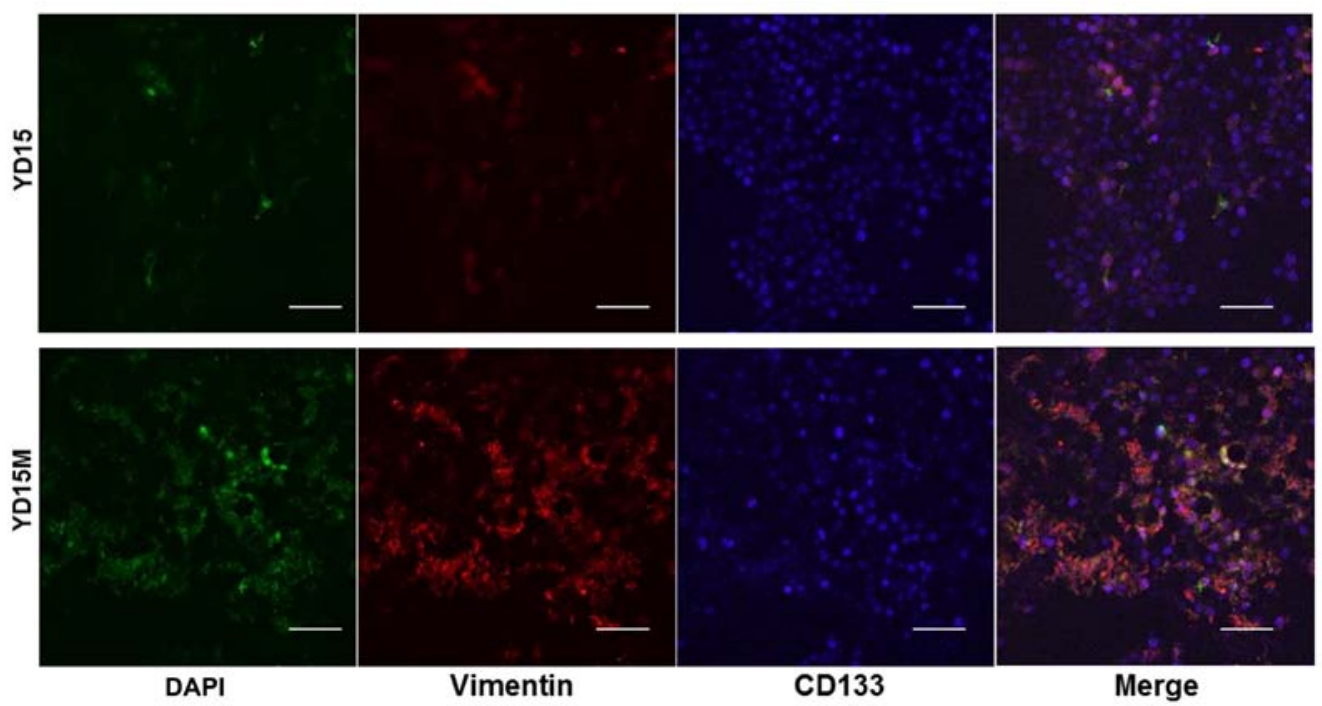

B
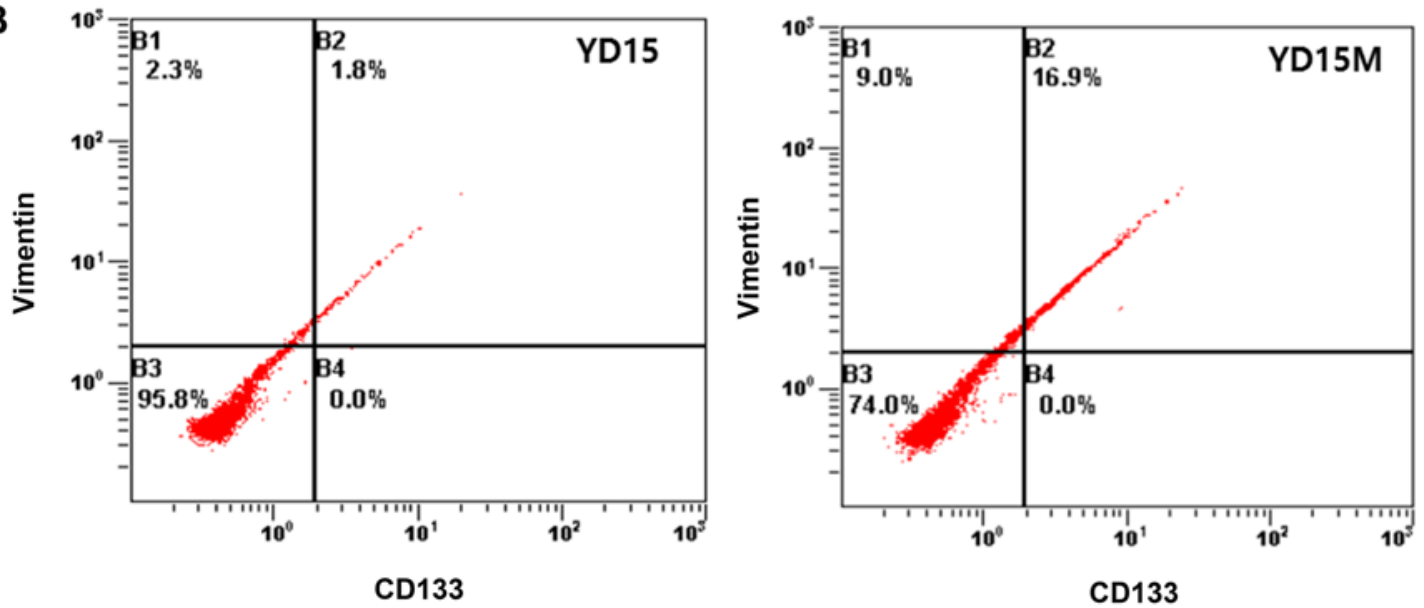

C
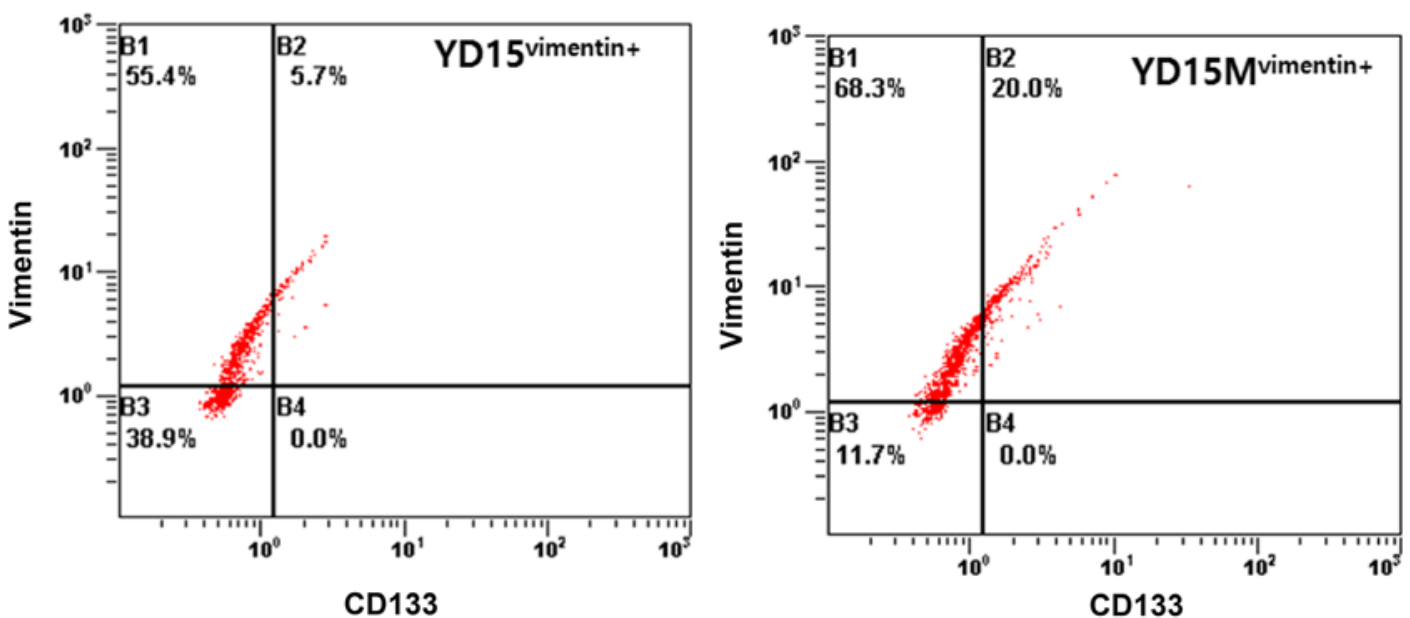

Figure 2. The relation of expression of the cancer stem cell marker CD133 with mesenchymal cell marker vimentin on primary cancer YD15 cells and metastatic YD15M cells. (A) Double immunofluorescence staining of vimentin (green) and CD133 (red). The nuclei in both image sets were stained with 4',6-diamidino2-phenylindole (DAPI; blue). Images were taken at x100 magnification. The scale bars are $100 \mu \mathrm{m}$. (B) The flow cytometric analysis of vimentin and CD133 expression in YD15 and YD15M cells (sector B2: coexpression). The corresponding mouse immunoglobulins conjugated to Texas Red (TR) or fluorescein isothiocyanate (FITC) were used as isotype controls in each experiment. (C) Flow cytometric analysis of vimentin and CD133 expression on vimentin ${ }^{+}$cells after magnetic cell separation (MACS).

properties corresponding to acquisition of CSC characteristics. To this end, we quantified the expression of CD133, PCNA, and CK by western blotting (Fig. 3A). Downregulation of PCNA, but not CD133, was observed in YD15M cells, in comparison with YD15 cells. In addition, there was no significant difference in CK expression between the two cell lines. In the dual-color 
A

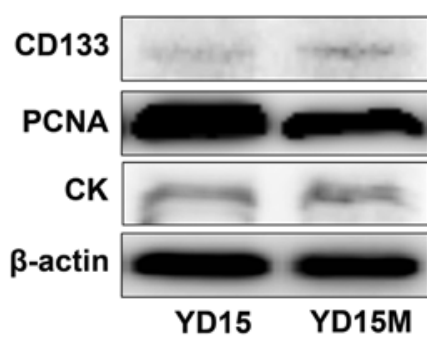

YD15

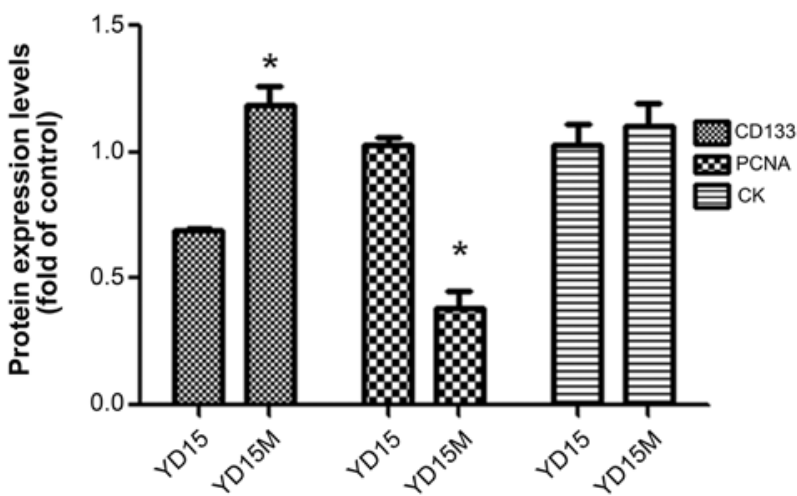

B
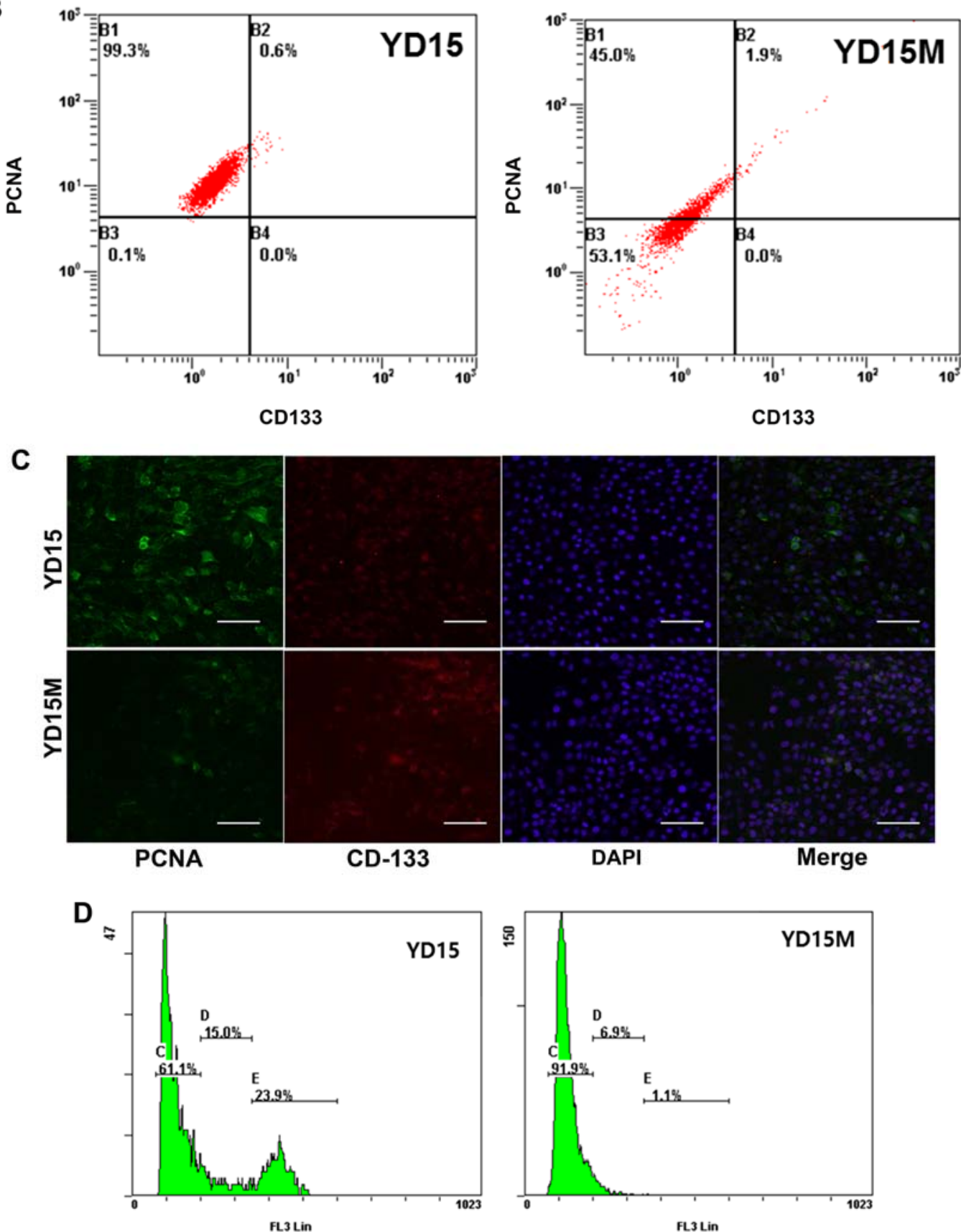

DAPI Merge

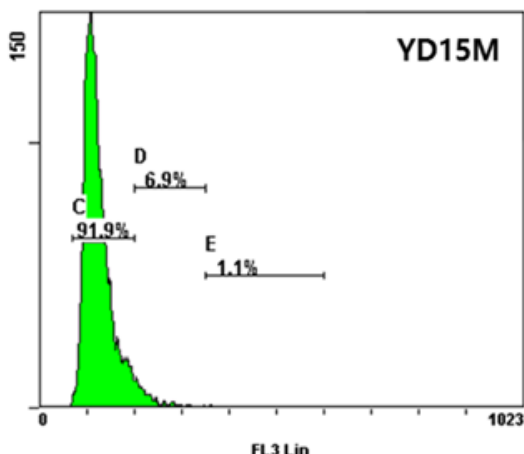

Figure 3. The relation of cell proliferative properties with expression of cancer stem cell marker CD133 on primary cancer YD15 cells and metastatic YD15M cells. (A) Western blot analysis of CD133, proliferative cell nuclear antigen (PCNA), and cytokeratin (CK). $\beta$-actin served as a loading control. The results were similar in 3 separate experiments and are presented as mean $\pm \mathrm{SD}$ (densitometry, as shown in the graphs). ${ }^{*} \mathrm{P}<0.05$, significant differences. (B) The flow cytometric analysis of PCNA and CD133 expression in the YD15 and YD15M cells. The corresponding mouse immunoglobulins [conjugated to Texas Red (TR) or fluorescein isothiocyanate; FITC] were used as isotype controls in each experiment. (C) Double immunofluorescence staining of PCNA (green) and CD133 (red). The nuclei in both image sets were stained with 4'6-diamidino-2-phenylindole (DAPI; blue); x100 magnification. The scale bars are $100 \mu \mathrm{m}$. (D) Cell cycle analysis of cell lines YD15 and YD15M. C means G0 and G1 phases; D, G2 and M phases; and E, S phase. 
A
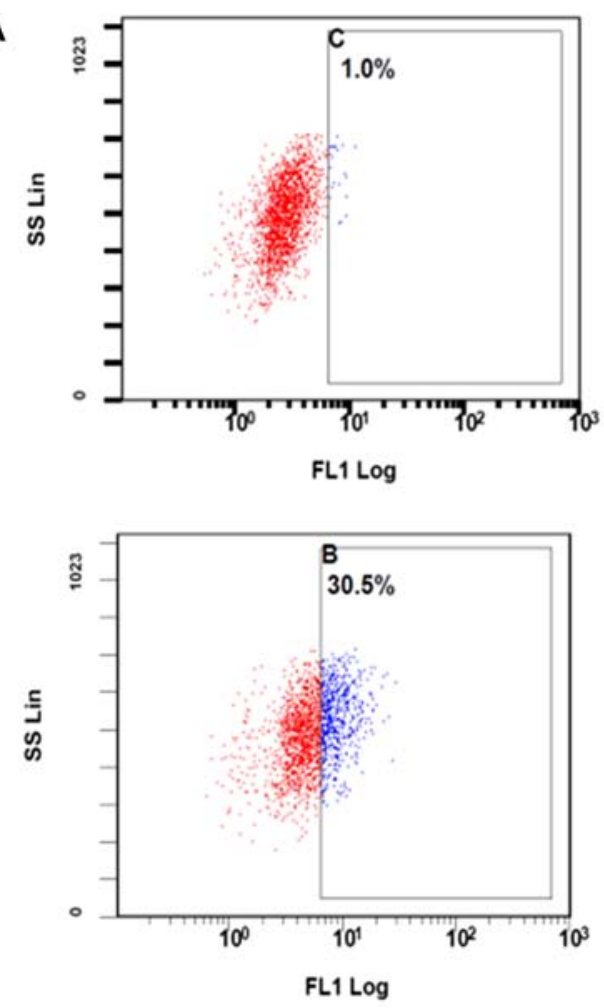

YD15
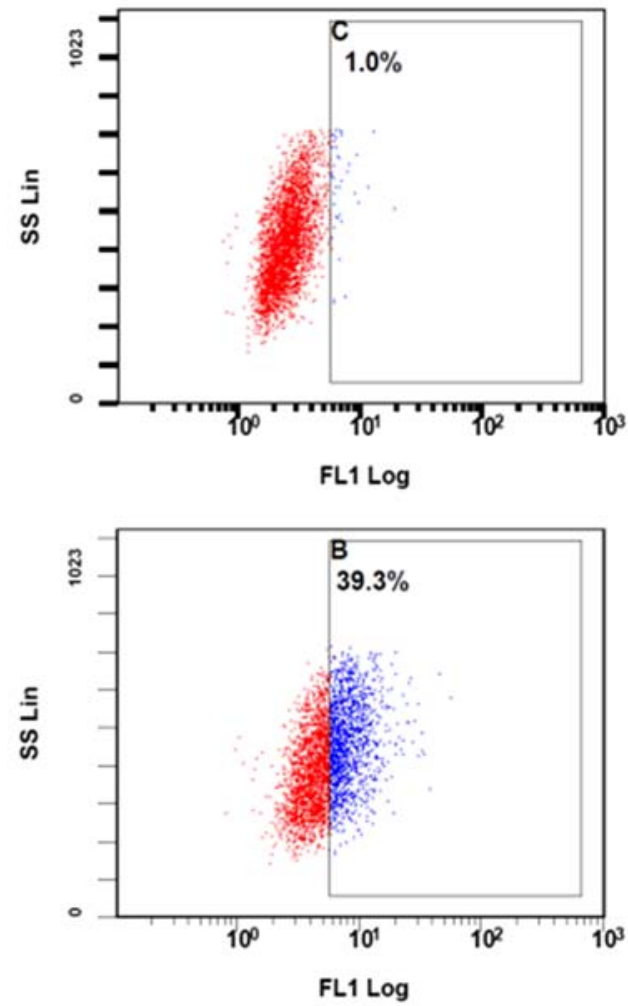

YD15M

B
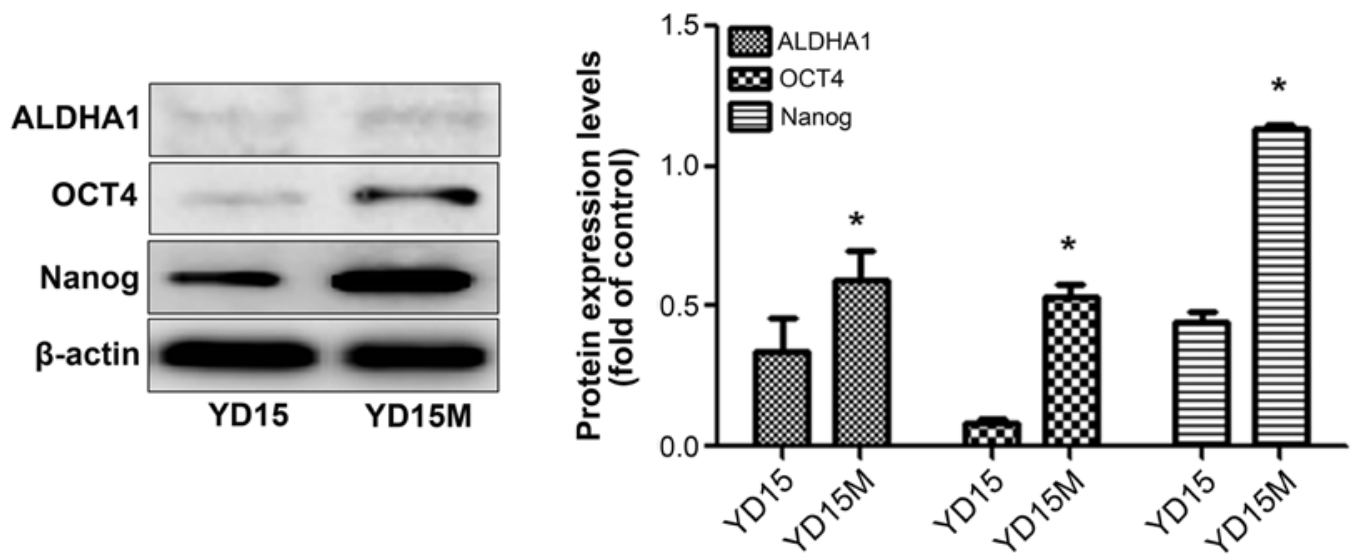

Figure 4. Examination of stem cell properties in the primary cancer cell line YD15 and metastatic YD15M cells. (A) Representative flow cytometry results are shown regarding aldehyde dehydrogenase (ALDH) activity. The first two columns at the top represent negative controls when the cells were treated with the ALDH inhibitor diethylaminobenzaldehyde (DEAB). The cells that are gated in sector C represent the subpopulation positive for ALDH activity. The two columns at the bottom represent the cells gated in sector B, the ALDH ${ }^{+}$population. (B) The levels of stemness-related proteins were analyzed by western blotting. $\beta$-actin served as a loading control. The results were similar in three separate experiments and are presented as mean \pm SD (densitometry, as shown in the graphs). ${ }^{*} \mathrm{P}<0.05$, significant differences.

flow cytometric analysis for both PCNA and CD133, simultaneous strong expression of PCNA and CD133 (gated in sector B2) could not detected among either YD15 or YD15M cells (Fig. 3B). As shown in the figure, the decreased expression of cell proliferation marker PCNA (green) in YD15M cells was not accompanied by downregulation of CD133 (red); this change corresponds to cells with increased CSC-like properties (Fig. 3C). To confirm proliferation indicators in YD15M cells (according to the PCNA western blotting), we performed cell cycle analysis (Fig. 3D). The population of YD15M cells contained a higher percentage of cells in the G0/G1 phase $(91.9 \%)$ than did the population of YD15 cells $(61.15 \%)$. In contrast, the total percentage of cells in the $\mathrm{S}$ phase was $1.1 \%$ among YD15M cells; this figure is lower than that among YD15 cells (23.9\%), confirming that YD15M cells had weak proliferative characteristics. Thus, we hypothesized that the weaker proliferation of the metastatic cell line YD15M may correspond to CSC-like properties.

Stem cell-like properties of primary cancer cells and metastatic cells. We utilized the ALDEFLUOR assay to assess the presence and size of the subpopulation with ALDH enzymatic activity among primary cancer cells and metastatic cells (Fig. 4A). The ALDEFLUOR-positive subpopulation consti- 
tuted $30.5 \%$ of YD15 cells. In contrast, it constituted $39.3 \%$ of YD15M cells. Furthermore, protein levels of ALDHA1, OCT4, and NANOG were higher in YD15M cells than in YD15 cells; in particular, OCT4 expression in YD15M cells was $>5$ times that in YD15 cells according to the western blot analysis (Fig. 4B). The increased ALDH activity and protein expression of ALDHA1, OCT4, and NANOG were suggestive of acquisition of stem cell-like properties during metastasis.

\section{Discussion}

Many patients with head and neck cancer die because of metastasis and/or recurrence of the tumor (30). Nevertheless, the main events underlying invasion and metastasis by cancer cells from the primary tumor are still unclear in head and neck cancer, although the link between EMT and metastasis was suggested recently $(8,31)$. One study suggested that progression of EMT endows cells with stem cell-like traits and allows them to become more invasive and able to migrate (32). Thus, we focused on the association of EMT with CSCs in primary cancer cells and metastatic cells; we tried to evaluate the relation between the expression of EMT markers and stem cell-like properties after metastasis.

EMT is an important step during the development of metastases of epithelial carcinoma; previous research provided in-depth understanding of the molecular mechanisms involved (8). The loss of E-cadherin, translocation of $\beta$-catenin to the nucleus, and induction of vimentin are crucial events during EMT in cancer cells (32). In this study, cancer cells showed morphological alterations from the typical cuboid epithelial phenotype to the mesenchymal phenotype and showed mesenchyme-like fibroblastic morphology accompanied by downregulation of the epithelial marker E-cadherin and upregulation of vimentin in the metastatic cell line YD15M. Partial nuclear localization of $\beta$-catenin was also observed. These results suggest that EMT processes take place during metastasis of mucoepidermoid carcinoma cells, as was reported in another study (33).

The theory on CSCs is still evolving but has gained momentum during the past five years; this theory can help to explain some of the key characteristics of specific tumors, including metastasis and the ability to invade (34). Recent studies support the notion that a tumor can be initiated and maintained by a small subpopulation of tumor cells that have stem cell-like properties, and these highly tumorigenic cells within the bulk of the tumor are considered CSCs (35).

In this study, we first sought to identify CSC-like cells using the cell surface marker CD133 (prominin 1). CD133 is a transmembrane glycoprotein (with five membrane-spanning domains) and was originally identified in neuroepithelial stem cells. CD133-expressing cancer cells with a stem cell-like phenotype are believed to account for tumor recurrence (36). Moreover, CD133 was found to be a common stemness marker and has been successfully used to isolate a subpopulation of highly tumorigenic cells from various solid tumors (37). In addition, CD133 is expressed by healthy-tissue resident and hematopoietic stem cells but is no longer detectable after differentiation. Similarly, CD133 is not expressed on cancer cells after their differentiation (25).
In this study, we showed that EMT is associated with acquisition of CSC properties. The protein levels of vimentin and CD133 were significantly higher in the metastatic cells than in the primary cancer cells. Furthermore, increased coexpression of vimentin and CD133 was observed in the metastatic cells. This result means that the population of vimentin ${ }^{+}$cancer cells acquired some properties of CSCs during metastasis.

In 2008, Mani et al reported that the induction of EMT in immortalized human mammary epithelial cells results in acquisition of mesenchymal traits and in expression of stem cell markers (38). CSC-like cells that are isolated either from mouse or human mammary glands or from mammary carcinomas also express EMT markers (38). The acquisition of these stem cell-like and tumorigenic characteristics is associated with EMT induction, and those results are in agreement with this study. These observations provide insights into the interplay between epithelial-mesenchymal plasticity and stem cell-like properties during metastasis and also cast doubt on the 'inevitable' association between EMT and CSCs. We believe that acquisition of CSC-like properties is accompanied by EMT and may stimulate migratory abilities of cancer cells.

Emerging evidence revealed a new characteristic of CSCs: their resistance to conventional chemotherapeutic agents because of the low proliferation rate (39). Here, we found that metastatic cells showed significantly lower expression of PCNA (cell proliferation marker) and a higher percentage of G0/G1 phase cells in the cell cycle. For this reason, metastatic cells are thought to have a slower proliferation rate, which may be one of CSC-like properties (40).

The crucial role of the pluripotency-related transcription factors (e.g., OCT4 and NANOG) in somatic cell reprogramming has been extensively studied (41). Nevertheless, the association of these factors with EMT in cancer cells is poorly understood. Sporadic reports link a gene signature of pluripotency to EMT; for example, most of these studies suggest that expression of pluripotency-related genes is associated with the mesenchymal phenotype and cancer invasiveness (42). In addition, some of these studies show that higher activity of ALDH (a cytosolic enzyme that is responsible for oxidation of intracellular aldehydes) can be used to improve identification of colon CSC population with the EpCAM ${ }^{\text {high }} \mathrm{CD} 44^{+}$phenotype as well as glioblastoma, retinoblastoma, and breast CSC populations $(43,44)$. In light of this study, it seems reasonable to hypothesize that expression of ALDH1A1, OCT4, and NANOG (or the more general marker: ALDH activity) may help to characterize CSCs during metastasis.

EMT was first recognized to be crucial for embryogenesis and is assumed to be required for invasiveness and metastasis of carcinoma cells because EMT promotes the loss of contact inhibition and increases cell motility. Therefore, the EMT pathway may be sufficient for acquisition of both invasiveness and stem cell properties by cancer cells; these changes will allow for establishment of a tumor at a distant site. We found that in addition to morphological changes, reduced E-cadherin expression and increased vimentin expression were associated with acquisition of EMT properties. In addition, coexpression of vimentin and CD133 was observed in metastatic cells, along with decreased PCNA expression, increased ALDH activity and upregulation of ALDHA1, OCT4, NANOG: these are CSC-like properties. These data substantiate the role of EMT 
and support the importance of CSCs during metastasis; therefore, our results could facilitate the development of a novel classification system and therapeutic strategies against oral cancer.

\section{Acknowledgements}

This study was supported by a grant (no. A121228) from the Korean Health Technology R\&D Project, Ministry of Health \& Welfare, Republic of Korea and the National Research Foundation of Korea grant funded by the Korean government (no. 2011-0030759).

\section{References}

1. Zhu LF, Hu Y, Yang CC, Xu XH, Ning TY, Wang ZL, Ye JH and Liu LK: Snail overexpression induces an epithelial to mesenchymal transition and cancer stem cell-like properties in SCC9 cells. Lab Invest 92: 744-752, 2012.

2. Sun L, Diamond ME, Ottaviano AJ,Joseph MJ, Ananthanarayan V and Munshi HG: Transforming growth factor-beta 1 promotes matrix metalloproteinase-9-mediated oral cancer invasion through snail expression. Mol Cancer Res 6: 10-20, 2008.

3. Silverman S Jr: Demographics and occurrence of oral and pharyngeal cancers. The outcomes, the trends, the challenge. J Am Dent Assoc 132 (Suppl): S7-S11, 2001.

4. Ramos DM, Dang D and Sadler S: The role of the integrin alpha $\mathrm{v}$ beta6 in regulating the epithelial to mesenchymal transition in oral cancer. Anticancer Res 29: 125-130, 2009.

5. Crnic I and Christofori G: Novel technologies and recent advances in metastasis research. Int J Dev Biol 48: 573-581, 2004.

6. Molloy T and van 't Veer LJ: Recent advances in metastasis research. Curr Opin Genet Dev 18: 35-41, 2008.

7. Yang CC, Zhu LF, Xu XH, Ning TY, Ye JH and Liu LK: Membrane Type 1 matrix metalloproteinase induces an epithelia to mesenchymal transition and cancer stem cell-like properties in SCC9 cells. BMC Cancer 13: 171, 2013.

8. Thiery JP: Epithelial-mesenchymal transitions in tumour progression. Nat Rev Cancer 2: 442-454, 2002.

9. Medema JP: Cancer stem cells: The challenges ahead. Nat Cell Biol 15: 338-344, 2013.

10. Charafe-Jauffret E, Ginestier C, Iovino F, Tarpin C, Diebel M, Esterni B, Houvenaeghel G, Extra JM, Bertucci F, Jacquemier J, et al: Aldehyde dehydrogenase 1-positive cancer stem cells mediate metastasis and poor clinical outcome in inflammatory breast cancer. Clin Cancer Res 16: 45-55, 2010.

11. Hermann PC, Huber SL, Herrler T, Aicher A, Ellwart JW, Guba M, Bruns CJ and Heeschen C: Distinct populations of cancer stem cells determine tumor growth and metastatic activity in human pancreatic cancer. Cell Stem Cell 1: 313-323, 2007.

12. Fan YL, Zheng M, Tang YL and Liang XH: A new perspective of vasculogenic mimicry: EMT and cancer stem cells (Review) Oncol Lett 6: 1174-1180, 2013.

13. Clarke MF, Dick JE, Dirks PB, Eaves CJ, Jamieson $\mathrm{CH}$ Jones DL, Visvader J, Weissman IL and Wahl GM: Cancer stem cells - perspectives on current status and future directions: AACR Workshop on cancer stem cells. Cancer Res 66: 9339-9344, 2006.

14. Elsaba TM, Martinez-Pomares L, Robins AR, Crook S, Seth R, Jackson D, McCart A, Silver AR, Tomlinson IP and Ilyas M: The stem cell marker CD133 associates with enhanced colony formation and cell motility in colorectal cancer. PLoS One 5: e10714, 2010

15. Bapat SA, Mali AM, Koppikar CB and Kurrey NK: Stem and progenitor-like cells contribute to the aggressive behavior of human epithelial ovarian cancer. Cancer Res 65: 3025-3029, 2005.

16. Hemmati HD, Nakano I, Lazareff JA, Masterman-Smith M, Geschwind DH, Bronner-Fraser M and Kornblum HI: Cancerous stem cells can arise from pediatric brain tumors. Proc Natl Acad Sci USA 100: 15178-15183, 2003.

17. Singh SK, Clarke ID, Terasaki M, Bonn VE, Hawkins C, Squire J and Dirks PB: Identification of a cancer stem cell in human brain tumors. Cancer Res 63: 5821-5828, 2003.
18. Al-Hajj M, Wicha MS, Benito-Hernandez A, Morrison SJ and Clarke MF: Prospective identification of tumorigenic breast cancer cells. Proc Natl Acad Sci USA 100: 3983-3988, 2003.

19. Collins AT, Berry PA, Hyde C, Stower MJ and Maitland NJ: Prospective identification of tumorigenic prostate cancer stem cells. Cancer Res 65: 10946-10951, 2005.

20. Fang D, Nguyen TK, Leishear K, Finko R, Kulp AN, Hotz S, Van Belle PA, Xu X, Elder DE and Herlyn M: A tumorigenic subpopulation with stem cell properties in melanomas. Cancer Res 65: 9328-9337, 2005.

21. Monroe MM, Anderson EC, Clayburgh DR and Wong MH: Cancer stem cells in head and neck squamous cell carcinoma. J Oncol 2011: 762780, 2011.

22. Davis SJ, Divi V, Owen JH, Bradford CR, Carey TE, Papagerakis S and Prince ME: Metastatic potential of cancer stem cells in head and neck squamous cell carcinoma. Arch Otolaryngol Head Neck Surg 136: 1260-1266, 2010.

23. Chang CC, Hsu WH, Wang CC, Chou CH, Kuo MY, Lin BR, Chen ST, Tai SK, Kuo ML and Yang MH: Connective tissue growth factor activates pluripotency genes and mesenchymalepithelial transition in head and neck cancer cells. Cancer Res 73: 4147-4157, 2013.

24. Nguyen PT, Kudo Y, Yoshida M, Iizuka S, Ogawa I and Takata T: $\mathrm{N}$-cadherin expression is correlated with metastasis of spindle cell carcinoma of head and neck region. J Oral Pathol Med 40: 77-82, 2011.

25. Mizrak D, Brittan M and Alison M: CD133: Molecule of the moment. J Pathol 214: 3-9, 2008.

26. Neuzil J, Stantic M, Zobalova R, Chladova J, Wang X, Prochazka L, Dong L, Andera L and Ralph SJ: Tumour-initiating cells vs. cancer 'stem' cells and CD133: What's in the name? Biochem Biophys Res Commun 355: 855-859, 2007.

27. Olempska M, Eisenach PA, Ammerpohl O, Ungefroren $\mathrm{H}$, Fandrich F and Kalthoff H: Detection of tumor stem cell markers in pancreatic carcinoma cell lines. Hepatobiliary Pancreat Dis Int 6: 92-97, 2007.

28. O'Brien CA, Pollett A, Gallinger S and Dick JE: A human colon cancer cell capable of initiating tumour growth in immunodeficient mice. Nature 445: 106-110, 2007.

29. Lim W, Kim J, Kim S, Karna S, Won J, Jeon SM, Kim SY, Choi Y, Choi $\mathrm{H}$ and Kim O: Modulation of lipopolysaccharideinduced NF- $\mathrm{KB}$ signaling pathway by $635 \mathrm{~nm}$ irradiation via heat shock protein 27 in human gingival fibroblast cells. Photochem Photobiol 89: 199-207, 2013.

30. Carvalho AL, Nishimoto IN, Califano JA and Kowalski LP: Trends in incidence and prognosis for head and neck cancer in the United States: A site-specific analysis of the SEER database. Int J Cancer 114: 806-816, 2005.

31. Kalluri R and Weinberg RA: The basics of epithelial-mesenchymal transition. J Clin Invest 119: 1420-1428, 2009.

32. Thiery JP, Acloque H, Huang RY and Nieto MA: Epithelialmesenchymal transitions in development and disease. Cell 139: 871-890, 2009.

33. Al Saleh S, Sharaf LH and Luqmani YA: Signalling pathways involved in endocrine resistance in breast cancer and associations with epithelial to mesenchymal transition (Review). Int J Oncol 38: 1197-1217, 2011.

34. Pang LY, Bergkvist GT, Cervantes-Arias A, Yool DA, Muirhead R and Argyle DJ: Identification of tumour initiating cells in feline head and neck squamous cell carcinoma and evidence for gefitinib induced epithelial to mesenchymal transition. Vet J 193: 46-52, 2012.

35. Zhou BB, Zhang H, Damelin M, Geles KG, Grindley JC and Dirks PB: Tumour-initiating cells: Challenges and opportunities for anticancer drug discovery. Nat Rev Drug Discov 8: 806-823, 2009.

36. Uchida N, Buck DW, He D, Reitsma MJ, Masek M, Phan TV, Tsukamoto AS, Gage FH and Weissman IL: Direct isolation of human central nervous system stem cells. Proc Natl Acad Sci USA 97: 14720-14725, 2000.

37. Iannolo G, Conticello C, Memeo L and De Maria R: Apoptosis in normal and cancer stem cells. Crit Rev Oncol Hematol 66: 42-51, 2008.

38. Mani SA, Guo W, Liao MJ, Eaton EN, Ayyanan A, Zhou AY, Brooks M, Reinhard F, Zhang CC, Shipitsin M, et al: The epithelial-mesenchymal transition generates cells with properties of stem cells. Cell 133: 704-715, 2008.

39. Tang X, Yao Y, Zhu J, Jin K, Wang Y, Mao Y and Zhou L: Differential proliferative index of cancer stem-like cells in primary and recurrent medulloblastoma in human. Childs Nerv Syst 28: 1869-1877, 2012. 
40. Anjomshoaa A, Nasri S, Humar B, McCall JL, Chatterjee A, Yoon HS, McNoe L, Black MA and Reeve AE: Slow proliferation as a biological feature of colorectal cancer metastasis. Br J Cancer 101: 822-828, 2009

41. Bourguignon LY, Wong G, Earle C and Chen L: HyaluronanCD44v3 interaction with Oct4-Sox2-Nanog promotes miR-302 expression leading to self-renewal, clonal formation, and cisplatin resistance in cancer stem cells from head and neck squamous cell carcinoma. J Biol Chem 287: 32800-32824, 2012.

42. Gangopadhyay S, Nandy A, Hor P and Mukhopadhyay A: Breast cancer stem cells: A novel therapeutic target. Clin Breast Cancer 13: 7-15, 2013.
43. Dalerba P, Dylla SJ, Park IK, Liu R, Wang X, Cho RW, Hoey T, Gurney A, Huang EH, Simeone DM, et al: Phenotypic characterization of human colorectal cancer stem cells. Proc Natl Acad Sci USA 104: 10158-10163, 2007.

44. Bar EE, Chaudhry A, Lin A, Fan X, Schreck K, Matsui W, Piccirillo S, Vescovi AL, DiMeco F, Olivi A, et al: Cyclopaminemediated hedgehog pathway inhibition depletes stem-like cancer cells in glioblastoma. Stem Cells 25: 2524-2533, 2007. 